\title{
Renormalization of Some One-Space Dimensional Quantum Field Theories by Unitary Transformation*
}

\author{
Paul Federbush \\ Department of Mathematics, Unirersity of Michigan, Ann Arbor, Michigan 48104 \\ Received May 4, 1970
}

The one-space dimensional $\varphi^{m} \bar{\psi} \psi$ interaction is renormalized by unitary transformation. A uniformly convergent sequence of unitary operators is detined which transforms the sequence of cut-off Hamiltonians, arranged in order of increasing cut-off energy, to a sequence of operators converging strongly on a dense set of states.

The theories here considered were treated in Ref. [1] by K. Hepp, using the method of J. Glimm [2]. We here treat these theories by use of methods initiated in [3]. We achieve an improvement of the result in [3] by obtaining a uniformly convergent sequence of unitary operators. The renormalization here performed is different from that in [1] and [2] in as much as unitary transformations are employed, but it is similar in that a form of truncation is used.

$$
H(\lambda)=H_{0}+V(\lambda)+\Delta(\lambda) .
$$

We will construct unitary operators $U(\lambda)$ with the property that

$$
\left.\left.\lim _{\lambda \rightarrow \infty} U^{-1}(\lambda) H(\lambda) U(\lambda) \mid \psi\right)=H \mid \psi\right)
$$

for $\psi \in \mathscr{D}$ a dense domain to be defined; the limit taken in a strong sense, and $H$ a symmetric operator.

Specifically, we consider the Hamiltonian

$$
H(\lambda)=H_{0 B}+H_{0 F}+V(\lambda)+\Delta(\lambda),
$$

with $\Delta(\lambda)$ the renormalization terms for

$$
V(\lambda)=g \int d x: \bar{\psi}_{\lambda}(x) \psi_{\lambda}(x):: \varphi_{\lambda}{ }^{\prime \prime}(x):
$$

* This work was supported in part by NSF grant GP 17523. 
The theory is constructed from fields defined on a unit interval with periodic boundary conditions:

$$
\begin{aligned}
H_{0 F} & =\sum_{p} \omega_{p}\left(a_{p}{ }^{*} a_{p}+b_{p}{ }^{*} b_{p}\right), \\
H_{0 B} & =\sum_{k} \mu_{k} d_{k}^{*} d_{k}^{\prime}, \\
\varphi_{\lambda}(x) & =\frac{1}{\sqrt{2}} \sum_{k} \frac{1}{\sqrt{\omega_{k}}} e^{i k \cdot x}\left(d_{k}+d_{-k}^{*}\right) \quad \omega_{k} \leqslant \lambda .
\end{aligned}
$$

As in [2], we define an operator $T^{N}$ that acts linearly on sums of normal ordered products of creation and annihilation operators:

$$
\begin{aligned}
& \Gamma^{N}\left(c_{p_{1}}^{*} \cdots c_{p_{s}}^{*} c_{p_{s+1}} \cdots c_{p_{T}}\right) \\
& = \begin{cases}p(N) \frac{1}{E_{p_{1}}+\cdots+E_{p_{s}}}-c_{p_{1}}^{*} \cdots c_{p_{T}} p(N) & \text { if } \sum_{1}^{s} \omega_{p_{i}}>\sum_{s+1}^{T} E_{p_{i}} \\
p(N) \frac{-1}{E_{p_{s+1}}+\cdots+E_{p_{T}}} c_{p_{1}}^{*} \cdots c_{p_{T}} p(N) & \text { if } \sum_{s+1}^{T} E_{p_{i}}>\sum_{1}^{s} E_{p_{i}} \\
0 & \text { if } \sum_{s+1}^{T} E_{p_{i}}=\sum_{1}^{s} E_{p_{i}}\end{cases}
\end{aligned}
$$

$c, c^{*}$ are understood as the $a, b, d, a^{*}, b^{*}, d^{*}$, and $E_{\eta_{i}}$ as either $\omega_{p_{i}}$ or $\mu_{p_{i}} \cdot p(N)$ is the projection onto states with $N$ or less particles in them. The use of $p(N)$ in the $\Gamma^{N}$ operation is the only truncation in the procedure to be followed.

The unitary operators $U(\lambda)$ are constructed as a product of unitary operators.

$$
U(\lambda)=\cdots e^{-A_{N}} \cdot e^{-A_{N-1}} \cdots e^{-A_{1}} .
$$

Here $A_{N}$ is constructed hy applying $\Gamma^{((M+2) N R)}$ to the sum of those terms in $V(\lambda)$ whose $E_{\max }$ satisfies

$$
\alpha N^{s}<E_{\max } \leqslant \alpha(N+1)^{s},
$$

$E_{\max }$ is the maximum energy of the creation and annihilation operators in a given term.

$$
E_{\max }\left(c_{p_{1}}^{*} \cdots c_{p_{s}}^{*} c_{p_{s+1}} \cdots c_{p_{T}}\right)=\max _{1 \leqslant i \leqslant T}\left(E_{p_{i}}\right)
$$

$\alpha, s$, and $R$ remain to be suitably chosen. 
The $L^{2}$ norm of the kernel of $A_{N}$ may be estimated as

$$
\sim c \sqrt{\frac{1}{\alpha N^{s}}-\frac{1}{\alpha(N+1)^{s}}} .
$$

Logarithmic powers are not relevant in this estimate, and with the projection operators $p((M+2) N R)$ appearing in $A_{N}$, we may estimate the operator norm of $A_{N}$

$$
\left|A_{N}\right| \sim c[(M+2) N R]^{\frac{M+2}{2}} \sqrt{\frac{1}{\alpha N^{s}}-\frac{1}{\alpha(N+1)^{s}}}
$$

if we satisfy jointly

$$
c^{2}[(M+2) R]^{M+2}=\alpha^{1-2 \beta},
$$

and

$$
s=\frac{M+3}{1-2 \beta}
$$

and

$$
1=s \beta^{2}
$$

It then follows that

$$
\left|A_{N}\right| \sim \frac{1}{\alpha^{\beta}} \frac{1}{N^{\beta S}}\left(\frac{\beta S}{N}\right)
$$

(15) and (16) determine $s$ and $\beta$, and (14) determines $\alpha$. It is to be noted that $R$ may be picked arbitrarily and only $\alpha$ effected.

Estimates are required for expressions such as

$$
U^{-1} H U=H+\sum_{R}\left(A_{R}, H\right)+\sum_{R_{1} \leqslant R_{2}}(!)\left(A_{R_{1}},\left(A_{R_{2}}, H\right)\right)+\cdots
$$

where (!) indicates the required products of factorials when indices are repeated. Estimates roughly proceed as

$$
\begin{aligned}
& \mid \sum_{R_{1} \leqslant R_{2}<\cdots \leqslant R_{s}}(!)\left(A_{R_{1}},\left(A_{R_{2}}, \ldots,\left(A_{R_{s}}, H\right) \cdots\right) \mid\right. \\
& \quad \leqslant \sum_{R_{1} \leqslant R_{2} \leqslant \cdots<R_{s}}(!) 2^{s}\left|A_{R_{1}}\right| \cdot\left|A_{R_{2}}\right| \cdots\left|A_{R_{2}}\right| \cdot|H| \\
& \quad \leqslant|H| \cdot e^{2 \Sigma\left|A_{R_{i}}\right|} .
\end{aligned}
$$


This estimate must be modified since $H$ has unbounded $L^{2}$ kernels to exploit the cancellations between the first few commutators. Because of the projections in the $A_{i}$ operators the cancellations not being exact, there are disconnected diagrams in the expansion (18). But if $R$ is chosen large enough, this causes no problems. It is exactly these disconnected terms that give difficulty in the $\left[\varphi^{4}\right]_{2+1}$ model. The domain $\mathscr{T}$ is chosen to consist of the states containing finite numbers of particles and whose particle momenta all lie in a bounded region. On this domain, the series (18) can be shown to converge using the operator norms of the $A_{i}$ above estimated, and estimates of the $L^{2}$ norm of the kernels of certain linear combinations of the first few commutators. It is not necessary either to count diagrams or to estimate the $L^{2}$ norm of arbitrarily complicated ones.

The computations here involved are simpler than those in [1] and [2]. Perhaps the unitary operator procedure will be useful for proving some properties of these models.

\section{REFERENCES}

1. K. HePp, Renormalized hamiltonians for a class of quantum fields with infinite mass and charge renormalizations, In "Essays in Honor of N. N. Bogoliubov," to appear.

2. J. Glimm, Comm. Math. Phys. 10 (1968), 1.

3. P. Federbush AND B. Gidas, Renormalization of the one-space dimensional Yukawa model by unitary transformation, 000 . 